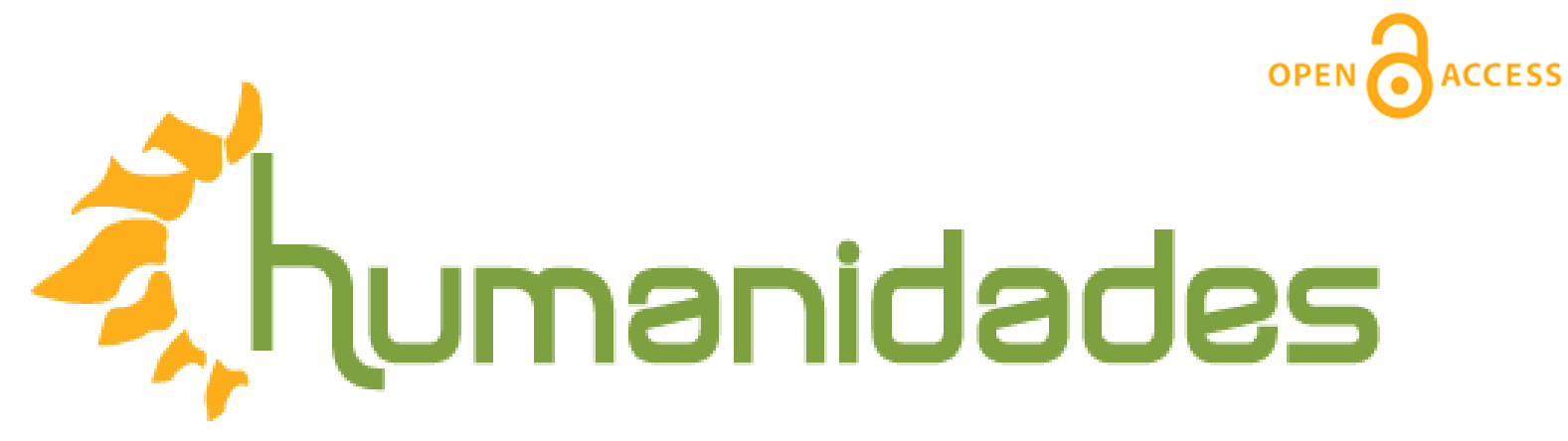

Revista de la Escuela de Estudios Generales, Universidad de Costa Rica

Julio-diciembre, 2016 • Volumen 6, número 2 • EISSN 2215-3934 • pp. 1-21

Recibido: 14-Abril-2016 Aceptado: 5-Julio-2016

\title{
La música: Un enfoque holístico del conocimiento
}

DOI: http://dx.doi.org/10.15517/h.v6i2.26743

\section{Margott Piedra Hernández}

Académica de la Escuela de Ciencias de la Educación de la Universidad Estatal a Distancia, específicamente, en el Programa de Licenciatura en Docencia, y directora del Coro de la Escuela de Ciencias de la Educación.

Correo electrónico: mpiedrah@uned.ac.cr

Todos los derechos reservados. Universidad de Costa Rica. Esta revista se encuentra licenciada con Creative Commons. Reconocimiento-NoComercial-SinObraDerivada 3.0 Costa Rica.

Correo electrónico: humanidades@ucr.ac.cr / Sitio web: http://revistas.ucr.ac.cr/index.php/humanidades 


\title{
La música: Un enfoque holístico del conocimiento
}

\section{Resumen}

A partir de la Ilustración, el mundo occidental comenzó a experimentar una fragmentación entre el saber y el conocimiento. Así, el pensamiento en el siglo XXI se debate entre esa división y los nuevos paradigmas en los que impera una posición holista de ver y entender el mundo y el conocimiento que se genera. No obstante, los modelos educativos continúan fortaleciendo postulados en los cuales el conocimiento tecnológico y científico adquiere más valor y relevancia, frente al saber ligado a las humanidades y a las artes. La música, íntimamente cohesionada con las matemáticas, los sentimientos y la física, representa el contexto ideal para entender que todo saber y toda creación humana forma parte de una totalidad, sin estructuras ni límites.

\section{Music: An holistic Approach of Knowledge}

\begin{abstract}
From the period of the Enlightenment the Western world began to experience a fragmentation of knowledge and understanding, thinking in the XXI century is torn between this division and new paradigms in the prevailing vision holistic view and understand the world and the knowledge that it is generated. Nevertheless, continue to strengthen educational models postulates that, technological and scientific knowledge becomes more valuable and relevant front that linked to the humanities and the arts know. The example of music, closely cohesive with math, feelings or physical, is the ideal to understand that all knowledge and all human creation is part of a whole, without structures or context limits.
\end{abstract}

Palabras claves: Música, conocimiento, filosofía, arte, acústica.

Keywords: Music, knowledge, philosophy, art, acoustics. 
En mayo de 1959, Charles Percy (1905-1980) dictó una Introducción conferencia sobre la polarización del conocimiento en Occidente: por un lado, el pensamiento humanista-social y, por otro, la ciencia. A propósito, Snow expone, en su ensayo Las dos culturas, la enorme brecha que surgió desde la Ilustración y que se acentuó en el siglo XIX, cuando en el periodo romántico se hicieron fuertes críticas, desde el "mundo intelectual", a los avances científicos que posibilitaron la Revolución Industrial y, particularmente, la sustitución del ser humano por la máquina.

En contraposición a lo comentado por Snow, el español José Pintado expone el concepto cultura transversal. Esta idea busca demostrar que la cultura es un sistema vivo que engloba al arte y a la ciencia. Además, Pintado manifiesta que el arte, la antropología, la historia, la ciencia y la tecnología son una construcción social, la cual, al generar conocimiento, puede transformar a la sociedad y transformarse a sí misma.

Pues bien, se puede decir que los avances de la ciencia han demostrado que el cerebro humano no procesa la información de manera fragmentada. De hecho, algunas investigaciones confirman que, a través de las emociones, se accede tanto al arte como al método científico. Sobre esto, Rodríguez, Rosado y Ramírez (2009) comentan que: 
Las definiciones más generales de ciencia la sitúan como un proceso social. ¿Es el arte a la vez, lo mismo? ... el arte ha estado estrechamente vinculado al conocimiento humano. Como expresión de la superestructura de la sociedad, contiene una esencia clasista al reflejar y promover la imagen e intereses de aquellos a quienes representa, subjetivada en diversas manifestaciones como modalidad de actividad humana. No toda creación humana puede ser arte pero todo arte es creación. De ello, no se aparta la ciencia (pp. 348-349).

En la actualidad, más de medio siglo después, el debate continúa. Lo anterior, principalmente, porque los sistemas educativos siguen fortaleciendo la idea de que el conocimiento se encuentra fragmentado: se encasilla en una u otra dirección. El modelo educativo costarricense no escapa de esta disputa, en gran medida, porque la laxa formación musical y artística, promovida desde el Estado, le asigna a la música una casi exclusiva función de ocio. Se le ubica, de acuerdo con Sánchez (2014), en “un estrato inferior en las artes (muy por debajo de la literatura o las artes plásticas)" (p. 1). Esta forma de entender y enseñar la música, entonces, pasa por alto siglos de tradición, puesto que en la antigüedad este arte ostentaba un significado más amplio que el otorgado en este momento y estaba íntimamente relacionado con la matemática y la astronomía. 
Así las cosas, este artículo representa un intento por demostrar que, durante miles de años, la música mantuvo una relación muy cercana con la ciencia. Además, se busca manifestar que la educación debe, desde un enfoque holístico del conocimiento, romper con la forma actual de ver y entender el saber y el mundo.

Pues bien, en el siglo VI a. C. los pueblos sumerios y sumitas advirtieron una estrecha relación entre la música y la matemática. Con la conquista helénica de Oriente Medio, la antigua Grecia heredó estos principios. Lo anterior se puede percibir en el pensamiento de Pitágoras, quien consideró que el sistema de sonidos y ritmos musicales responde a un orden numérico, el cual se encuentra al servicio de la compresión del universo espiritual y físico.

Este filósofo griego, al observar las vibraciones que producían las cuerdas de algunos instrumentos musicales, como la lira y la kithara (conocida en América Latina como cítara), logró teorizar sobre la propuesta antes planteada. De esa forma, propuso llamar a una cuerda vibrante L; al formarse una doble longitud de cuerda (2L) se crea un intervalo de octava. Bajo estos principios, 3/2L establece un intervalo de quinta justa y 4/3L constituiría un intervalo de cuarta. Dichas relaciones fueron representadas mediante las siguientes proporciones matemáticas: $(2: 1,3: 2,4: 3 \ldots)$. 
Los principios pitagóricos de las proporciones matemáticas, relaciones geométricas y música, alcanzan a otros fenómenos de la naturaleza; así pues, a cada movimiento se le asigna un sonido particular. El movimiento de los planetas, por ejemplo, produciría intervalos musicales en el universo, lo cual originaría una especie de polifonía planetaria.

Más adelante, Platón (427-347 a. C.) retoma la relación entre música, matemática y astronomía, para explicar la combinación de sonidos del universo. Nace, entonces, la teoría de la música de las esferas, según la cual los movimientos de los astros originan sonidos; en consecuencia, los planetas producen acordes armónicos perceptibles solo a través del intelecto.

En su obra La República, Platón describe el mito del guerrero Er, quien al morir ve el universo. $\mathrm{Al}$ respecto, menciona que las órbitas de los planetas formaban acordes emitidos por una sirena: "Encima de cada uno de los círculos iba una sirena que daba también vueltas y lanzaba una voz siempre del mismo tono; y de todas las voces, que eran ocho, se formaba un acorde" (Platón, 2011, p. 186).

La idea perduró durante toda la Edad Media, época en la que incluso "se llegó a creer que los diez mandamientos formaban una especie de dedacorde, que el número tres era el más perfecto en 
música, o que toda la música podría derivarse del número doce" (Torres, 2009, p. 104).

En el siglo XVII, René Descartes (1596-1650), en su obra Compendio de Música (1618), retoma la idea de las vibraciones armónicas que producen los instrumentos de cuerda. Sobre esto, menciona que la cuerda vibra en varias ondas que son múltiplos de una nota fundamental. Dicho pensamiento matemático-musical posibilitó la generación de nuevas teorías, estilos musicales y formas de afinar los instrumentos; es así como nace la escala cromática. Esta última consiste en una escala de doce semitonos que permiten, entre otros asuntos, cambiar de tonalidad en una misma obra, sin tener que afinar nuevamente los instrumentos (modulación).

Precisamente el uso de la escala cromática o temperada facilita apreciar cómo la música y la ciencia están íntimamente relacionadas. A propósito, el matemático y religioso francés Marin Mersenne (1588-1648), creador de los números primos de Mersenne, formuló las reglas usadas hasta hoy para afinar cualquier instrumento, en las cuales se utiliza la siguiente fórmula: $\mathrm{Fi}=440 \times 2$ i/12.

La explicación de fórmula anterior no forma parte de los objetivos de este artículo. Basta con entender que la afinación de los instrumentos musicales occidentales toma como base la frecuencia 
de la vibración a $440 \mathrm{~Hz}$, lo cual corresponde a la nota La. Es decir, hablar de música es hablar de frecuencias, resonancias, vibraciones, intervalos, propagación del sonido, entre otros.

Así lo entendió el astrónomo y matemático alemán Johannes Kepler (1571-1630), quien retomó la teoría de la música de las esferas de Platón. Kepler argumenta que las velocidades del movimiento de los planetas operan las mismas proporciones utilizadas en la música. En ese sentido, cuanto más rápido sea el movimiento de un astro, más agudo será su sonido (perihelio); en tanto que, una menor velocidad generará un sonido grave (afelio). Por consiguiente, cada planeta experimenta, por causa de su desplazamiento, melodías que se generan en el lapso de un día, en los puntos extremos de su órbita (afelio y perihelio).

El movimiento celeste no es otra cosa que una continua canción para varias voces, para ser percibida por el intelecto, no por el oído; una música que, a través de sus discordantes tensiones, a través de sus síncopas y cadencias progresa hacia cierta predesignada cadencia para seis voces, y mientras tanto deja sus marcas en el inmensurable flujo del tiempo (Kepler; citado por González, 2005, párr. 9). 
Ahora bien, las leyes del movimiento de Isaac Newton (1643-1727) desvirtuaron la teoría de la música de las esferas por casi doscientos años. Durante ese tiempo, se consolidó la idea de que la ciencia y el arte estaban separadas por la razón. En esto pesó, sobre todo, la imagen que surgió en el romanticismo (1800-1890), donde la música responde a un sentido de creatividad "divina" en la que interviene únicamente la emoción frente a la razón. De hecho, con frecuencia se asociaba la creación artística "con estados anormales de neurosis; es decir crean los locos" (Flores, 2004, p. 20).

A partir de ese momento, muchos intentaron demostrar, con visiones bastante reduccionistas, que existe una división entre científicos y literatos; sin embargo, desde finales del siglo XIX, una serie de descubrimientos científicos han acercado cada vez más al arte y a la ciencia. Por ejemplo, el hallazgo de la desexitación del átomo develó que cada átomo produce rayos de emisión que se expresan en una fórmula de números enteros, similar a la de los intervalos musicales.

En el siglo XX, la Administración Nacional de la Aeronáutica y del Espacio (NASA) confirma que la atmósfera del Sol produce sonidos ultrasónicos, los cuales interpretan una melodía formada por ondas trecientas veces más graves que el tono que puede ser percibido por el oído humano. Incluso, teorías recientes de la Física describen las 
partículas más elementales como vibraciones de pequeñas cuerdas que, como dijo Pitágoras, se plasman en simetrías matemáticas.

En adelante, las teorías interdisciplinarias y holísticas para entender al mundo (y el conocimiento que en este se crea) han tomado importancia. Así, en los últimos treinta años se han realizado estudios para tratar de entender la mágica relación entre el pensamiento musical y científico. Por ejemplo, el psiquiatra y neurólogo Robert S. Root-Bernstein (1953), profesor de fisiología en la Universidad de Michigan, Estados Unidos, comprobó que las habilidades musicales y científicas están correlacionadas; para ello, analizó el pensamiento y obra de importantes científicos como Albert Einstein y Richard Feynman (considerado uno de los científicos estadunidenses más importantes del siglo XX y ganador del Premio Nobel de Física en 1965).

Lo dicho anteriormente permitió comprobar que existen personas capaces de sentir y entender, de una forma multimodal y sintética, la música y la intuición científica. Al respecto, Root-Berstein y Root-Berstein mencionan: "Incluso los poetas más verbales y científicos matemáticos sostienen que la creación de su obra emerge de sentimientos, emociones e imágenes sensuales" (2003, p. 6). El mismo Einstein afirmaba "con frecuencia pienso en la música y sueño despierto con ella. Veo mi vida en forma de música". 
Todos estos hallazgos que permiten comprender la relación entre música y ciencia se fundamentan también en la historia de la creación musical propiamente dicha, ya que la relación físicamatemática-música ha interesado a numerosos compositores. Por ejemplo, Wolfang Amadeus Mozart (1756-1791), en 1777, crea la obra Juego de dados musical para escribir valses con la ayuda de dos dados sin ser músico ni saber nada de composición (K294); en esta resulta interesante el hecho de que no fue escrita en una partitura, sino en un sistema numérico que depende del azar.

El juego de composición inicia al lanzar los dados. Cada "compositor" debe arrojarlos ocho veces, lo cual resulta en una serie de posibilidades de valses, de dieciséis compases cada uno. El mismo Mozart escribió 176 compases y los agrupó en dos tablas de 88 elementos numéricos cada una. A partir de mediciones de álgebra superior, se descubrió que el número de valses posibles es de $11^{16}$, es decir, para escuchar todas las posibilidades de composiciones se necesitan 361 millones de años.

En el siglo XX, numerosos compositores han mostrado interés por las propiedades del número en la composición, en un intento por alcanzar la objetividad y pureza en la música. Es así como el compositor y matemático Joseph Schillinger (1895-1943) desarrolló un sistema de composición musical basado en la 
matemática. Dicho sistema fue descrito en su libro Mathematical Basis of the Arts (1943):

"The fundamental components of music, viz., scales, cadences, triads, sevenths, etc., have been transformed into their coherent natural geometric form, in color and in $3 \mathrm{rd}$ dimensión. This enables us to visually analyze these musical structures as well as their mirror images, thus dissolving the inherent bewilderment we all encounter in our music studies. It also accelerates the learning process by a thousand fold by enabling us to see the general overall mechanics of music" [Los componentes fundamentales de la música, a saber, escalas cadencias, tríadas, séptimos, etc., se han transformado en su forma geométrica natural, coherente, en color y en tercera dimensión. Esto nos permite analizar visualmente estas estructuras musicales, así como sus imágenes especulares, disolviendo así la perplejidad inherente que todos encontramos en nuestros estudios de música. También acelera el proceso de aprendizaje por mil veces por lo que nos permite ver los mecanismos generales de la música] (Lyden, 1996, párr. 2).

Schillinder, a su vez, mostró gran fascinación por las posibilidades de composición que ofrece la serie numérica de Fibonacci. Esta 
serie, que responde a la siguiente fórmula $(F n+2=F n+1+F n(n \geq 0)$, consiste en una sucesión de números donde cada nuevo número es el resultado de la suma de los números anteriores, a saber: 0, 1, 1, 2, $3,5,8,13,21,34,55,89,144 \ldots$ De manera que las obras compuestas, según estos principios, presentan notas sucesivas de la melodía siguiendo intervalos al estilo de esta serie numérica.

No obstante, las relaciones son más profundas; la serie Fibonacci, por ejemplo, también guarda relación con el número áureo, representado por los griegos mediante $\varphi$. En la Edad Media, el matemático y teólogo Luca Pacioli asoció este número con el principio divino universal de la belleza. En téminos matemáticos, la proporción áurea o número de oro se define como la relación que guardan dos segmentos de recta entre sí. Dicha relación puede evidenciarse en la pintura, la escultura y la arquitectura, tal es el caso de El Hombre de Vitrubio, de Leonardo da Vinci; y la obra del artista alemán Alberto Durero, conocida como Espiral de Durero, la cual describe la espiral áurea.

En la música, las representaciones no son tan obvias. Los casos más citados son la Sinfonía N. 5 de Ludwig van Beethoven, las obras de Franz Schubert, Debussy y Béla Bartók. En la música de Bartók, la proporción áurea y la serie Fibonacci se aprecian en el ritmo y en la 
melodía, por ejemplo, en la alternación de compases de 8 pulsos con compases de 5 pulsos.

Otro ejemplo, más accesible para el lector que no conoce la obra de estos músicos, es el teclado de un piano moderno. Lo anterior porque cada octava tiene 8 teclas blancas y 5 teclas negras (alteraciones) que aparecen en grupos de 2 y 3 ; y la serie 2, 3, 5, 8 es el inicio de la serie numérica de Fibonacci.

A mediados del siglo $\mathrm{XX}$, nuevos métodos de composición continúan fundamentándose en el sustento científico de la música. Muestra de esto es el dodecafonismo o música atonal, un sistema musical que se sustenta en la igualdad de los doce tonos de la escala cromática, lo cual forma series ordenadas de notas para la creación de las composiciones. Este sistema se amplió posteriormente en el serialismo integral, cuyos exponentes (Olivier Messiaen, Pierre Boulez, Karlheinz Stockhausen y Luigi Nono) crearon una corriente musical fuertemente determinada por la matemática, al establecer series ordenadas ya no solo de tonos (como el dodecafonismo), sino también de ritmos y dinámicas.

Puede mencionarse, además, el caso de la música estocástica, la cual utiliza las leyes del cálculo, la teoría de conjuntos, las leyes de probabilidades y la lógica simbólica para crear música 
indeterminada. Así mismo, la música espectral que se produce por medio de las representaciones y análisis de los espectros del sonido: espectro armónico formado por las frecuencias de los tonos graves y agudos que son múltiplos enteros de la nota fundamental. Otro ejemplo es la composición a partir de fractales, cuyos principios descansan en la proyección del comportamiento dinámico de un fractal sobre un espacio musical. Para comprender este último estilo es pertinente aclarar que un fractal es una figura semi geométrica formada por componentes infinitos; en la música esto se representa con sonidos que se repiten de acuerdo con patrones de comportamiento espontáneos.

Todos estos nuevos estilos musicales llevan a que algunos compositores, como Babbitt, afirmen que la sociedad debe considerar las innovaciones artísticas tal y como se consideran las innovaciones científicas. Aunado a esto, se exige que el éxito de una obra musical no se debe medir en función de la aceptación del público, ni de la industria del entretenimiento.

El siglo XX, asimismo, posibilitó que ciertas teorías derivadas del desarrollo del pensamiento de la Física ejercieran una gran influencia en el progreso de la música. Ejemplos de lo anterior son la teoría de la incertidumbre y la teoría de la relatividad, las cuales se proyectan en la música indeterminada de John Cage y en la 
música aleatoria del alemán Karlheinz Stockhausen, Alberto Ginastera, entre otros.

Estos avances en la composición inevitablemente han facilitado el surgimiento de contribuciones tecnológicas; la música electrónica es un claro ejemplo. En definitiva, el desarrollo musical de la mano de la tecnología ha permitido un mayor conocimiento acerca del sonido, de hecho, se descubre su utilidad en la bioquímica y en la medicina. En este campo se encuentran los estudios del Dr. Susumo Ohno, quien ha convertido las cadenas del ADN en secuencias musicales. Susumo afirma que los seres humanos tienen mayor capacidad para captar las modificaciones melódicas, en comparación con las numéricas y visuales.

En esa línea, el fisiólogo Hugh Lusted le asignó valores a los movimientos de los músculos del cuerpo humano y los transformó en señales eléctricas. Luego, estos se convirtieron en sonidos musicales que se ejecutan con un instrumento electrónico bautizado por el mismo Lusted como biomusa. Todos estos descubrimientos podrían, en el futuro, colaborar en la detección de disfunciones y enfermedades psiquiátricas.

Así las cosas, lo expuesto hasta ahora permite vislumbrar que la música es más que notas, casualidad, inspiración y sentimientos. 
Antes bien, reside en este arte una inalienable propiedad científica que recuerda la transversalidad del conocimiento; una realidad en la que el arte es cultura, pero la ciencia también. Puesto que la cultura no es una creación individual sino social, la ciencia y el arte también son humanidades.

La educación, por lo tanto, debe encargarse de develar las fronteras imaginarias y autoimpuestas del conocimiento. En ese sentido, establecer un modelo educativo con visiones epistemológicas amplias, para comprender que "El conocimiento humano, o es transversal o no es conocimiento, ni humano" (Arsuaga, 2015, s. p.).

Consecuentemente, la enseñanza debe centrar su atención en potenciar y respetar a la música y a la educación artística en general. De manera que se logre "resignificar su postura científica, y consolidarla como disciplina de integración sociocultural... y no dispuesta en parcelas separadas... que en su devenir han sido eclipsados por la afluencia de modelos manufacturados y mecanicistas enclavados en contenidos cientificistas" (Pérez, 2012, p. 7).

Este nuevo paradigma atenderá los principios de integralidad expuestos desde el humanismo, superando las visiones reduccionistas de los currículos que aún permean las prácticas socioeducativas costarricenses. Al respecto, Magendzo (2003) recuerda que "Lo que 
se pretende hoy es transformar la visión utilitarista de la sociedad, de la cultura como una "trama" externa a la escolarización y al curriculum, es decir, caracterizar el contexto escolar dentro de las "necesidades" y los objetivos sociales deseados a los que la educación debe responder (p. 29).

La música ha acompañado al ser humano desde la aparición de los primeros homínidos. Así, de forma casi imperceptible para la mayoría, ha estado íntimamente relacionada con el desarrollo del conocimiento científico y tecnológico. Desgraciadamente, la herencia occidental europea del Siglo de las luces separó ambas ramas del saber, relegando a la música, y en general a las artes, a actividades de ocio y placer; al tiempo que asoció la creación musical con estados divinos de inspiración, relacionados con conceptos como la creatividad.

Ante esta división del conocimiento, algunos, desde nuevas posturas epistemológicas, defienden que el arte y la ciencia proceden de una misma indagación del ser humano frente al mundo. Lo anterior significa que "ninguna cosa o hecho existe de manera independiente de una trama de articulaciones y conexiones... cada parte trae en sí el todo, reflejando la unicidad del universo. Es donde arte y ciencia pueden interrelacionarse de manera inesperada" (Pérez, 2010, p. 19). 
Si se quiere avanzar en el desarrollo de una cultura transversal, se debe entender que entre la música (el arte en general) y la ciencia existen más que conexiones casuales; en realidad, coexisten interrelaciones e intersecciones que las potencian. En palabras de Pérez (2010), se trata de una "relación tridimensional" (p. 41), en la cual cada parte se superpone a la otra.

En suma, el debate planteado por Snow, hace más de 50 años, parece tener vigencia, principalmente porque el ser humano insiste en ver y conceptualizar el conocimiento desde una imagen única. Parece, entonces, que la tarea pendiente de la historia, de la cultura y, fundamentalmente, de la educación es entender, desde la transversalidad, la complejidad del conocimiento. 
De la Iglesia, F., Loeck, J., Caeiro, J. y Martín, R. (Eds.). (2010). La cultura transversal. Colaboraciones entre arte, ciencia y tecnología. Vigo: Universidad de Vigo.

García, R. (2009). La teoría de la armonía de las esferas en el libro quinto de Harmonices Mundi de Johannes Kepler (Tesis de maestría). Universidad de Salamanca, España.

González, N. (2005). Música de les esferes. Universitat Pompeu Fabra. Recuperado de https://www.upf.edu/pdi/dcom/xavierberenguer/recursos/fig_calc/_4_/ estampes/3_13.htm

Kaufmann, T. (2011). Arte y conocimiento: rudimentos para una perspectiva descolonial. Instituto europeo para políticas culturales. Recuperado de http://eipcp.net/transversal/0311/kaufmann/es

Lyden, H. (1996-1994). The Schillinger System of Musical Composition [El sistema Schillinder de composición musical]. Recuperado de http://www.schillingermusic.com/

Magendzo, A. (1986). El currículum y cultura en América Latina. Santiago, Chile: Programa Interdisciplinario de investigación en educación.

Magendzo, A. (1991). Currículum y Cultura en América Latina. Santiago, Chile: Programa interdisciplinario de investigación en educación.

Moure, G. (2003). El síndrome de Mozart. Madrid: Ediciones SM.

Peretz, I. (2006). Brain Specialization for Music: New Evidence from Congenital Amusia. Annals, 930(1), 153-165.

Platón, (2011). La República. Buenos Aires, Argentina: Tecnibook Ediciones. 
Rodríguez, S., Rosado, R. M. y Ramírez, M. R. (2009). Las dos culturas de C. P. Snow. Un acercamiento crítico desde el oficio del antropólogo. Ra Ximhai, 5(3), 347-355. Recuperado de http://www.uaim.edu.mx/webraximhai/Ej15articulosPDF/09\%20Las_dos_culturas.pdf

Root-Bernstein, R. y Root-Bernstein, M. (2003). Intuitive Tools for Innovative Thinking. En L. Shavinina (Ed.), The International Handbook on Innovation (pp. 377-387). Oxford: Elsevier Science.

Sánchez, L. (2011). Relaciones entre música, ciencia y tecnología durante el siglo XX. Encuentros multidisciplinares, 13(38), 1-10. Recuperado de http://www.encuentrosmultidisciplinares.org/Revistan\%BA38/Leticia_S\%E1nchez_de_Andr $\%$ E9s.pdf

Torres, J. (2009). La música como ciencia. Revista de arte y estética contemporánea. Recuperado de http://www.saber.ula.ve/bitstream/123456789/30943/1/articulo10.pdf

Universidad de Burgos. [tvUBU Universidad de Burgos]. (2015, Febrero 25). Conferencia "El conocimiento humano, o es transversal o no es conocimiento, ni humano". [Archivo de video]. Recuperado de https://www.youtube.com/watch?v=V_zSdl3y1iA

\section{¿Cómo citar este artículo?}

Piedra, M. (Julio-diciembre, 2016). La música: Un enfoque holístico del conocimiento. Revista humanidades, 6(2), 1-21. doi: dx.doi.org/10.15517/h.v6i2.26743 